\title{
Profiling of HLA-B Alleles for Association Studies with Ankylosing Spondylitis in the Chinese Population
}

\author{
Lin $\mathrm{Yi}^{1,2, \S}$, Jiucun Wang ${ }^{3,5,}$, Xinjian Guo ${ }^{1}$, Maribel G. Espitia ${ }^{1}$, Enuo Chen ${ }^{1}$, Shervin Assassi ${ }^{1}$, \\ Li Jin $^{3}$, Hejian Zou ${ }^{*, 4,5}$, John D. Reveille ${ }^{1}$ and Xiaodong Zhou ${ }^{*}, 1$ \\ ${ }^{I}$ Division of Rheumatology and Clinical Immunogenetics, University of Texas Medical School at Houston, USA \\ ${ }^{2}$ Gansu College of Traditional Chinese Medicine, Lanzhou, Gansu, China \\ ${ }^{3}$ State Key Laboratory of Genetic Engineering and Ministry of Education Key Laboratory of Contemporary \\ Anthropology, School of Life Sciences, Fudan University, China \\ ${ }^{4}$ Huashan Hospital, Fudan University, China \\ ${ }^{5}$ Institute of Rheumatology, Immunology, and Allergy, Fudan University, China
}

\begin{abstract}
Human leucocyte antigen (HLA) B*27 is a susceptibility allele to ankylosing spondylitis (AS). However, major AS-associated subtypes of HLA-B*27 and other HLA-B alleles vary in different ethnic populations. Herein, we examined HLA-B alleles in a total of 360 AS patients and 350 controls of Chinese Han ancestry. The HLA-B genotyping was performed with sequence-based typing (SBT) method. Six HLA-B*27 subtypes B*27:04, B*27:05, B*27:07, B*27:08, $B * 27: 10$ and $B * 27: 15$ were observed in the cohorts. HLA-B*27:04:01 and -B*27:05:02 appeared significantly increased in AS patients, which indicated as two major susceptibility alleles to AS. Homozygous $B * 27$ was observed only in AS patients. There are 30 HLA-B alleles identified in the studies. HLA-B*15, especially $\mathrm{B}^{*} 15: 01: 01: 01$, appeared as the major allele type in the Chinese controls. Some common HLA-B alleles such as HLA-B*15, B*13, B*46 and B*51 were significantly reduced in Chinese AS patients. In conclusion, the studies profiled the HLA-B alleles, and identified major susceptibility subtypes of B27 to AS in Han Chinese population.
\end{abstract}

Keywords: Ankylosing spondylitis (AS), HLA-B27, Chinese Han.

\section{INTRODUCTION}

Ankylosing spondylitis (AS) is an immune-mediated chronic disease characterized by inflammation of the axial skeleton, as well as extraspinal involvement. Although, etiopathogenesis is not fully understood, genetic factors play a key role in AS susceptibility. The human leukocyte antigen (HLA) class I molecules are essential in the immune regulation, especially in defense against intracellular infections (e.g. viruses). In fact, representing the wide array of infectious challenges facing humans, HLA alleles have evolved as the most polymorphic loci in the genome, with over 6919 MHC class I and 1875 class II alleles having been reported as of January, 2013 (http://www.ebi.ac.uk/ipd/ imgt/hla/stats.html), many of which conferring susceptibility to specific immune-mediated diseases.

\footnotetext{
*Address correspondence to these authors at the Division of Rheumatology and Clinical Immunogenetics, University of Texas Medical School at Houston, 6431 Fannin Street, Room 5.270 Houston, Texas 77030, USA; Tel: 713-500-6900; Fax: 713-500-0580;

E-mail:xiaodong.zhou@uth.tmc.edu and Huashan Hospital, Fudan University, China; Tel: +86-21-52888808; Fax: +86-21-62489191; E-mail: hejianzou@gmail.com
}

${ }^{\S}$ First two authors contributed to the studies equally.
HLA-B is a class I molecule, and HLA-B27 is a welldocumented genetic risk allele for ankylosing spondylitis (AS) in all examined populations, which contributes $23.3 \%$ of AS heritability [1]. However, of the over $100 \mathrm{~B} * 27$ alleles reported to date, the major AS-associated subtypes of HLAB27 vary in frequency in different ethnic populations. HLA$B * 27: 02$ was reported in AS patients of Europeans, especially more frequent in Southern European [2]. HLA$B * 27: 03$ appeared in Black African populations [3]. HLA$B * 27: 04$ is the most common AS-risk allele in Chinese Han, Taiwanese and Japanese [4-6], HLA-B*27:05, the "parent" HLA-B27 allele, is present in almost all populations, and is the major AS allele in Caucasians, American Indians [3] and Koreans [7]. HLA-B*27:06 is a relatively rare subtype of HLA-B27 occurring most commonly in Southeast Asian individuals, Thai, Taiwanese and Singaporean populations [8], and is thought to be unassociated with AS. Similarly, the presence of HLA-B*27:07 and HLA-B*27:08 were reported less frequently in AS patients. HLA-B*27:07 may play a protective role [9]. HLA-B*27:09 described only in Sardinia and southern Italy [10], and has not been observed to data in AS patients.

Although, several studies of HLA-B have been reported in Chinese AS patients, most of them examined only $B * 27$ positive individuals. $\mathrm{B} * 27$ negative information is generally lack in the studies. Recently, we established an AS cohort 
and controls of Han Chinese. Our studies aimed to profile the HLA-B alleles, and to determine major and minor HLA$\mathrm{B}$ alleles, as well as whether they are potential risk or protective alleles to AS in Han Chinese.

\section{MATERIALS AND METHODOLOGY}

\section{Patients and Controls}

Han Chinese patients and controls were enrolled from the clinics and hospitals in Shanghai and Jiangsu Province of China. A total of 360 AS patients and 350 controls of Chinese Han ancestry were examined in the studies. All patients met the modified New York criteria for this disease [11]. All participants underwent a clinical evaluation by one of the study rheumatologists, had pelvic and spinal radiographs to confirm their diagnosis, and completed questionnaires about their personal and medical history and functional status. Unrelated controls were free of any history of rheumatic disease. Chinese controls were obtained from a study project of Chinese population genetics in Fudan University, Shanghai, China. All subjects signed the informed consent. The studies were approved by Ethic Committee of both Fudan University and the University of Texas Medical School at Houston.

\section{Sequencing Genotyping}

Genomic DNA was extracted from peripheral blood cells from subjects. The HLA-B genotyping was performed with sequence-based typing (SBT) method using SeCore Kits (Life Technologies, USA). Briefly, the allele-specific polymerase chain reactions (PCR) were performed using primers supplied in the SeCore kits, and then were followed by sequencing exon 2 and 3 of the HLA-B gene. The HLA SBT UTYPE 6.0 program (Life Technologies) was used in sequencing analysis and assigning HLA-B alleles.

\section{Data Analysis}

Tests of association between genetic variants and AS were performed for each allele using Epi Info program developed by the Center for Disease Controls and Prevention (CDC. Atlanta, Georgia, USA). Exact p-values were obtained (Fisher's test) from $2 \times 2$ tables of allele counts and disease status.

\section{RESULTS}

Sequencing data were first filtered with quality control according to the HLA SBT uTYPE 6.0 program. The samples that failed in sequencing were re-examined. There were six AS patients and eight controls who failed in genotyping although the sequencing quality passed the filter. A total of 354 AS patients and 342 controls were successfully genotyped.

There were thirty HLA-B alleles identified in the studies (Table 1). HLA-B*15, especially B*15:01:01:01, appeared as the most common allele in controls $(31.3 \%$ controls carrying $\mathrm{B}^{*} 15$ with allele frequency of $17.5 \%$ ). HLA-B*40, HLA-B*13 and HLA-B*46 also appeared as common alleles of Chinese controls with corresponding allele frequencies of $12.8 \%, 11.4 \%$ and $10 \%$ respectively (Table 1). Statistical results for each of HLA-B alleles are listed in the Table 1.
Table 1. Allele Frequency of HLA-B in Han Chinese AS Cases and Controls

\begin{tabular}{|c|c|c|c|c|c|c|}
\hline Alleles & AS & $\%$ & Control & $\%$ & $p$ & OR \\
\hline $\mathrm{B} * 07$ & 1 & 0.1 & 12 & 1.8 & 0.0018 & 0.08 \\
\hline B*08 & 2 & 0.3 & 5 & 0.7 & 0.237 & 0.38 \\
\hline $\mathrm{B}^{*} 13$ & 39 & 5.5 & 78 & 11.4 & $7.4 \times 10^{-5}$ & 0.45 \\
\hline $\mathrm{B} * 14$ & 3 & 0.4 & 1 & 0.2 & 0.334 & 2.91 \\
\hline $\mathrm{B} * 15$ & 51 & 7.2 & 120 & 17.5 & $<1 \times 10^{-7}$ & 0.36 \\
\hline $\mathrm{B} * 18$ & 2 & 0.3 & 1 & 0.2 & 0.584 & 1.93 \\
\hline $\mathrm{B} * 27$ & 336 & 47.5 & 26 & 3.8 & $<1 \times 10^{-7}$ & 22.86 \\
\hline $\mathrm{B} * 35$ & 14 & 2.0 & 37 & 5.4 & $6.6 \times 10^{-4}$ & 0.35 \\
\hline$B * 37$ & 2 & 0.3 & 4 & 0.6 & 0.389 & 0.48 \\
\hline$B * 38$ & 12 & 1.7 & 20 & 2.9 & 0.126 & 0.57 \\
\hline $\mathrm{B} * 39$ & 15 & 2.1 & 10 & 1.5 & 0.356 & 1.46 \\
\hline$B * 40$ & 73 & 10.3 & 88 & 12.9 & 0.136 & 0.78 \\
\hline$B * 41$ & 0 & 0 & 1 & 0.1 & 0.309 & 0 \\
\hline$B * 44$ & 9 & 1.3 & 16 & 2.3 & 0.134 & 0.54 \\
\hline$B * 45$ & 0 & 0 & 7 & 1.0 & 0.007 & 0 \\
\hline $\mathrm{B} * 46$ & 36 & 5.1 & 68 & 10 & $5.7 \times 10^{-4}$ & 0.49 \\
\hline$B * 48$ & 12 & 1.7 & 11 & 1.6 & 0.899 & 1.05 \\
\hline $\mathrm{B} * 50$ & 2 & 0.3 & 0 & 0 & 0.164 & NA \\
\hline $\mathrm{B} * 51$ & 29 & 4.1 & 60 & 8.8 & $3.6 \times 10^{-4}$ & 0.44 \\
\hline $\mathrm{B} * 52$ & 12 & 1.7 & 19 & 2.8 & 0.171 & 0.6 \\
\hline $\mathrm{B} * 53$ & 1 & 0.1 & 0 & 0 & 0.325 & NA \\
\hline B*54 & 10 & 1.4 & 19 & 2.8 & 0.075 & 0.5 \\
\hline $\mathrm{B} * 55$ & 12 & 1.7 & 14 & 2.1 & 0.628 & 0.83 \\
\hline$B * 56$ & 1 & 0.1 & 4 & 0.6 & 0.167 & 0.24 \\
\hline$B * 57$ & 4 & 0.6 & 4 & 0.6 & 0.96 & 0.97 \\
\hline $\mathrm{B} * 58$ & 25 & 3.5 & 50 & 7.3 & 0.0018 & 0.46 \\
\hline B*59 & 0 & 0 & 4 & 0.6 & 0.042 & 0 \\
\hline B*67 & 3 & 0.4 & 3 & 0.4 & 0.966 & 0.97 \\
\hline $\mathrm{B} * 78$ & 1 & 0.1 & 2 & 0.3 & 0.543 & 0.48 \\
\hline B*81 & 1 & 0.1 & 0 & 0 & 0.325 & NA \\
\hline
\end{tabular}

A $p$-value less than 0.00167 is significant after correction for multiple comparison.

HLA-B $* 27$ occurred in $93 \%$ in AS patients and $7.6 \%$ of Han Chinese controls. There were six subtypes of the HLA$\mathrm{B} * 27(\mathrm{~B} * 27: 04, \mathrm{~B} * 27: 05, \mathrm{~B} * 27: 07, \mathrm{~B} * 27: 08, \mathrm{~B} * 27: 10$ and $\left.\mathrm{B}^{*} 27: 15\right)$ observed in Chinese AS patients, only three of them $\left(\mathrm{B}^{*} 27: 04, \mathrm{~B}^{*} 27: 05\right.$ and $\left.\mathrm{B} * 27: 07\right)$ occurred in the controls (Table 2). Among the six subtypes, B*27:04 (or $\mathrm{B} * 27: 04: 01)$ appeared in $68.1 \%$ AS patients versus in $4.1 \%$ controls, and $\mathrm{B}^{*} 27: 05: 02$ in $22 \%$ AS patients versus $2.63 \%$ controls. HLA-B*27:07:01, B*27:08, B*27:10 and B*27:15 were sporadically observed in the AS patients. There were nine AS patients homozygous for $\mathrm{B} * 27$ alleles, of whom four were homozygous for $B * 27: 04: 01$. In contrast, all controls with $\mathrm{B} * 27$ positive were heterozygous with a non- 
$\mathrm{B} * 27$ allele $(\mathrm{p}=0.0033)$. Specific allele frequencies in cases and controls and their statistical results are listed in the Table 2.

Table 2. Presence of the HLA-B27 Subtypes (Allele Frequency) in All Chinese AS Patients and Controls

\begin{tabular}{|c|c|c|c|c|c|c|}
\hline Alleles & AS & $\%$ & Control & $\%$ & $p$ & OR \\
\hline \multicolumn{7}{|l|}{ B*27:04 } \\
\hline$B * 27: 04: 01$ & 245 & 34.6 & 16 & 2.3 & $<1 \times 10^{-7}$ & 22.09 \\
\hline \multicolumn{7}{|l|}{$B * 27: 05$} \\
\hline$B * 27: 05: 01$ & 1 & 0.1 & 0 & 0 & 0.325 & NA \\
\hline$B * 27: 05: 02$ & 79 & 11.2 & 9 & 1.3 & $<1 \times 10^{-7}$ & 9.42 \\
\hline$B * 27: 07$ & 5 & 0.7 & 1 & 0.2 & 0.111 & 4.86 \\
\hline$B * 27: 08$ & 1 & 0.1 & 0 & 0 & 0.325 & NA \\
\hline$B * 27: 10$ & 1 & 0.1 & 0 & 0 & 0.325 & NA \\
\hline$B * 27: 15$ & 4 & 0.6 & 0 & 0 & 0.049 & NA \\
\hline Total B alleles & 708 & & 684 & & & \\
\hline
\end{tabular}

\section{DISCUSSION}

The genetic association of HLA-B*27 with AS was discovered first in the early 1970's, and was persistently confirmed in later studies. However, specific subtypes of HLA-B27 and major susceptibility alleles of the subtypes to AS vary in different ethnic populations. Specific alleles and subtypes of HLA genes are named according to their corresponding serologic antigen and the complexity of gene sequence. In general, the first set of digits (first two digits) corresponds to the serological antigen carried by an allotype. The second set of digits (second two digits) is used to name the subtypes that are nonsynonymous nucleotide substitutions. The third set of digits (third two digits) is assigned to synonymous nucleotide substitutions, the fourth set of digits (fourth two digits) indicate specific sequence polymorphisms in non-coding regions of the gene.

In Chinese population, HLA-B*27:04 was reported as the major susceptibility allele in AS patients, and was followed by HLA-B*27:05 [12-14]. In addition to these two subtypes, $\mathrm{B} * 27: 02, \mathrm{~B} * 27: 03, \mathrm{~B} * 27: 06, \mathrm{~B} * 27: 10, \mathrm{~B} * 27: 13, \mathrm{~B} * 27: 15$ and B27:24 also were reported in Chinese AS patients. The results of our studies indicated that HLA-B*27:04 primarily and $\mathrm{B} * 27: 05$ secondarily were the most highly associated AS alleles, consistent with previous reports. Our results specified these two alleles of subtypes with the third set of digits as $\mathrm{B} * 27: 04: 01$ and $\mathrm{B} * 27: 05: 02$. In addition to these two alleles, $\mathrm{B} * 27: 07, \mathrm{~B} * 27: 08, \mathrm{~B} * 27: 10$ and $\mathrm{B} * 27: 15$ also were observed in our AS patients. Among them, $B * 27: 07$ $(B * 27: 07: 01)$ and $B * 27: 08$ are reported first time in Chinese AS patients, although their allele frequencies were only $0.7 \%$ and $0.1 \%$, respectively (Table 2 ). However, a protective role of $\mathrm{B} * 27: 07$ against AS that was reported previously [9] cannot be confirmed here. A larger sample size of studies may be necessary to clarify this association. Another important feature of this report is that homozygous $\mathrm{B} * 27$ was observed in $2.54 \%$ AS patients, but was absent in controls, which supports a risk role of $\mathrm{B} * 27$ to Chinese AS.
In contrast to HLA-B*27 as the major allele in Chinese AS patients, HLA-B*15 was observed in $31.3 \%$ Chinese controls as the most common allele (allele frequency of $17.5 \%$ ). Specifically, $B * 15: 01: 01: 01$ was the major subtype of $\mathrm{B}^{*} 15$ in the population. HLA-B*13, HLA-B*40 and HLA-B*46 also appeared as common alleles of Chinese controls (allele frequency $\geq 10 \%$ ) (Table 1). HLA-B*13, $B * 15$ and $B * 46$ were significantly decreased in the patients indicating their potential protective role against AS of Chinese population. There were a total of 30 HLA-B alleles identified in the studies, which provided a profile of Chinese HLA-B alleles.

In addition to HLA-B*27 with AS, HLA-B*14:03 also was associated with AS in a West African population [15], however, it was not observed in Chinese population in our studies. It is worth noting that various HLA-B alleles have been implicated in association with other disease susceptibility and clinical outcomes. For instance, HLA$B * 51$ has been recognized as genetic susceptibility factor for Behcet's disease (BD) [16], HLA-B*44 was reported to protect against multiple sclerosis (MS) [17], HLA-B*58:01 and $\mathrm{B}^{*} 15: 02$ were associated with allopurinol-induced and carbamazepine-induced Stevens-Johnson syndrome and toxic epidermal necrolysis, respectively [18-20], HLA$B * 57: 01$ was considered as a major determinant of druginduced liver injury due to flucloxacillin [21]. Our profiling of HLA-B alleles of Chinese population provides a reference for future studies of different diseases in association with specific HLA-B alleles.

\section{CONCLUSION}

Our studies profiles the HLA-B alleles, and identified HLA-B*27:04:01 and $B * 27: 05: 02$ as two major susceptibility suballeles of HLA-B*27 to AS in Han Chinese population. We also demonstrated that homozygous HLA$B * 27$ only occurred in AS patients which may suggest strong risk to AS. Some common alleles of Chinese population including $\mathrm{B}^{*} 15, \mathrm{~B} * 13$ and $\mathrm{B} * 46$ are significantly reduced in Chinese AS patients, which may implicate potentially protective role to AS.

\section{CONFLICT OF INTEREST}

The authors confirm that this article content has no conflict of interest.

\section{ACKNOWLEDGEMENTS}

This study was supported by the NIH NIAID 1U01AI090 90-01, the Major National Science and Technology Program of China, grant number 2008ZX10002-002, and the Science and Technology Committee of Shanghai Municipality (114 10701800).

\section{REFERENCES}

[1] Brown MA. Progress in the genetics of ankylosing spondylitis. Brief Funct Genomics 2011; 10: 249-57.

[2] Reveille JD, Maganti RM. Subtypes of HLA-B27: history and implications in the pathogenesis of ankylosing spondylitis. Adv Exp Med Biol 2009; 649: 159-76.

[3] Ben Radhia K, Ayed-Jendoubi S, Sfar I, et al. Distribution of HLA$B * 27$ subtypes in Tunisians and their association with ankylosing spondylitis. Joint Bone Spine 2008;75: 172-5.

[4] Liu Y, Jiang L, Cai Q, et al. Predominant association of HLAB*2704 with ankylosing spondylitis in Chinese Han patients Tissue Antigens 2010; 75: 61-4. 
[5] Hou TY, Chen HC, Chen CH, Chang DM, Liu FC, Lai JH. Usefulness of human leucocyte antigen-B27 subtypes in predicting ankylosing spondylitis: Taiwan experience. Intern Med J 2007; 37 : 749-52.

[6] Yamaguchi A, Ogawa A, Tsuchiya N, et al. HLAB27 subtypes in Japanese with seronegative spondyloarthropathies and healthy controls. J Rheumatol 1996; 23: 1189-93.

[7] Sung-Hoon P, JiHun K, Sang-Gyung K, Seong-Kyu K, Won TC, Jung-Yoon C. Human leucocyte antigen-B27 subtypes in Korean patients with ankylosing spondylitis: higher $\mathrm{B} * 2705$ in the patient group. Int J Rheum Dis 2009; 12: 34-8.

[8] Lopez-Larrea C, Sujirachato K, Mehra NK, et al. HLA-B27 subtypes in Asian patients with ankylosing spondylitis. Evidence for new associations. Tissue Antigens 1995; 45: 169-76.

[9] Varnavidou-Nicolaidou A, Karpasitou K, Georgiou D, et al. HLAB27 in the Greek Cypriot population: distribution of subtypes in patients with ankylosing spondylitis and other HLA-B27-related diseases. The possible protective role of $B * 2707$. Hum Immunol 2004; 65: 1451-4.

[10] Paladini F, Taccari E, Fiorillo MT, et al. Distribution of HLA-B27 subtypes in Sardinia and continental Italy and their association with spondylarthropathies. Arthritis Rheum 2005; 52: 3319-21.

[11] Van der Linden S, Valkenburg HA, Cats A. Evaluation of diagnostic criteria for ankylosing spondylitis. A proposal for modification of the New York criteria. Arthritis Rheum 1984; 27: 361-8.

[12] Zhen W, Yikun M, Zhiming L, Jinxian H, Qiujing W, Jieruo G. HLA-B27 polymorphism in Han Chinese patients with ankylosing spondylitis: a distinctive disease association for $\mathrm{B} * 2715$ in a multiplex family. J Rheumatol 2009; 36: 2849-50.
[13] Zhang L, Liu JL, Zhang YJ, Wang H. Association between HLAB*27 polymorphisms and ankylosing spondylitis in Han populations: a meta-analysis. Clin Exp Rheumatol 2011; 29: 28592.

[14] Liu X, Hu LH, Li YR, Chen FH, Ning Y, Yao QF. The association of HLA-B*27 subtypes with ankylosing spondylitis in Wuhan population of China. Rheumatol Int 2010; 30: 587-90.

[15] López-Larrea C, Mijiyawa M, González S, et al. Association of ankylosing spondylitis with HLA-B*1403 in a West African population. Arthritis Rheum 2002; 46: 2968-71.

[16] Kaburaki T, Takamoto M, Numaga J, et al. Genetic association of HLA-A*2601 with ocular Behçet's disease in Japanese patients. Clin Exp Rheumatol 2010; 28: S39-44.

[17] Healy BC, Liguori M, Tran D, et al. HLA B*44: protective effects in MS susceptibility and MRI outcome measures. Neurology 2010; 75: 634-40.

[18] Hung SI, Chung WH, Liou LB, et al. HLA-B*5801 allele as a genetic marker for severe cutaneous adverse reactions caused by allopurinol. Proc Natl Acad Sci USA 2005; 102: 4134-9.

[19] Tassaneeyakul W, Jantararoungtong T, Chen P, et al. Strong association between HLA-B*5801 and allopurinol-induced Stevens-Johnson syndrome and toxic epidermal necrolysis in a Thai population. Pharmacogenet Genomics 2009; 19: 704-9.

[20] Zhang Y, Wang J, Zhao LM, et al. Strong association between HLA-B*1502 and carbamazepine-induced Stevens-Johnson syndrome and toxic epidermal necrolysis in mainland Han Chinese patients. Eur J Clin Pharmacol 2011; 67: 885-7.

[21] Daly AK, Donaldson PT, Bhatnagar P, et al. DILIGEN Study; International SAE Consortium. HLA-B*5701 genotype is a major determinant of drug-induced liver injury due to flucloxacillin. Nat Genet 2009; 41: 816-9.

() Yi et al.; Licensee Bentham Open.

This is an open access article licensed under the terms of the Creative Commons Attribution Non-Commercial License (http://creativecommons.org/licenses/by-nc/ 3.0/) which permits unrestricted, non-commercial use, distribution and reproduction in any medium, provided the work is properly cited. 